Stomatologie $2015 \cdot 112: 1$

DOI 10.1007/s00715-015-0013-1

Online publiziert: 6. März 2015

(c) Springer-Verlag Wien 2015

\title{
Gerhard Kreyer
}

Langenlois, Österreich

\section{Psychologie und Psychosomatik in der Zahnmedizin}

versuchen bzw. chirurgischen Eingriffen hinter sich.

Daher kommt adäquaten diagnostischen Überlegungen und therapeutischen Konzeptionen auch aus zahnärztlicher Sicht eine besondere Bedeutung zu. scher Sicht in besonderer Weise als Projektionsfeld seelischer Konflikte oder psychischer Erkrankungen prädestiniert.

Konfrontationen mit sog. Problempatienten haben ihre Ursachen zumeist nicht in zahnärztlich-fachspezifischen Problemstellungen, sondern in einer Störung der Arzt-Patient-Interaktion.

Gerade der Bereich des Mundes und der Zähne mit z. T. sehr kostenaufwendigen Sanierungen bietet sich als Manifestationsorgan für sog. Somatisierungen innerpsychischer Probleme auf dem Weg einer somatischen orofazialen Schiene an.

Dazu gehören einerseits Ängste, die sich teilweise überdeutlich erkennbar, teilweise aber auch in maskierter Form präsentieren.

Andererseits finden sich unterschiedlichste Symptombilder, funktionelle Störungen oder Somatisierungen in Hinblick auf die Psychosomatik des Bezahnten, die Psychosomatik des Unbezahnten und die Psychosomatik des Zahnverlustes. Problemkreise wie „psychogene Amalgamintoleranz" und „somatoforme Prothesenunverträglichkeit" zählen ebenso dazu wie orale Habits, orofaziale Parafunktionen, das „Burning-Mouth-Syndrom“, psychosomatische Probleme bei drogenabhängigen Patienten oder das „psychodentale Begleitsyndrom“.

Charakteristischerweise haben viele Patienten aufgrund von Defiziten in der Primärversorgung und präliminären Diagnostik eine lange und komplizierte Anamnese mit zahlreichen negativen Untersuchungen und ergebnislosen somatischen wie auch „alternativen“ Therapie-

\section{》) Belastungen bedürfen adäquater Bewältigungsstrategien}

In der zahnmedizinischen und dentalpsychologischen Literatur werden Schwierigkeiten zwischen Arzt und Patient zum weit überwiegenden Teil in Hinblick auf den Patienten diskutiert. Sehr viel weniger Aufmerksamkeit wird der Problematik des Zahnarztes selbst gewidmet.

Es bedarf aus psychohygienischer Sicht adäquater Bewältigungsstrategien, mit den Belastungen - bis hin zum sog. Burnout-Syndrom -, die sich für den Zahnarzt und im weiteren Sinne auch für sein Team aus der Konfrontation mit Problempatienten ergeben, umzugehen.

Das vorliegende Themenheft über „Psychologie und Psychosomatik in der Zahnmedizin“ bietet Beiträge von diagnostischer und therapeutischer Relevanz für die tägliche Arbeit in der zahnärztlichen Praxis ebenso wie solche für die Coping-Kompetenz und Psychohygiene des Behandlers selbst.

\section{Ihr G. Kreyer}

Vorsitzender der Österreichischen Gesellschaft für Psychologie und Psychosomatik in der Zahnmedizin

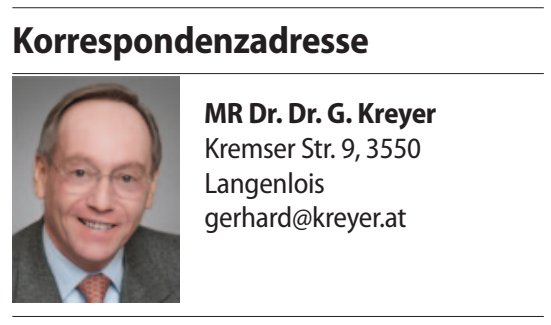

\section{Einhaltung ethischer Richtlinien}

Interessenkonflikt. G. Kreyer gibt an, dass kein Interessenkonflikt besteht.

Dieser Beitrag beinhaltet keine Studien an Menschen oder Tieren. 